\title{
Inscription of Fiber Bragg Grating Arrays in Pure Silica Suspended Core Fibers
}

\author{
Martin Becker, Luis Fernandes, Manfred Rothhardt, Sven Brückner, Kay Schuster, Jens Kobelke, \\ Orlando Frazão, Hartmut Bartelt, and Paulo V. S. Marques
}

\begin{abstract}
Fiber Bragg grating (FBG) arrays in pure silica fourleaf-clover-shaped suspended core fibers were inscribed with twobeam interference and a deep UV femtosecond laser source. The target fibers are pure silica and do not contain any dopants, nor were they treated with hydrogen before grating inscription. The inscription method is appropriate to measure the effective mode field index of the fiber and allows confinement factors of the guided modes to be derived. Applications of such FBGs can be expected especially for fiber sensing.
\end{abstract}

Index Terms-Femtosecond laser, fiber Bragg gratings (FBGs), optical fibers, photosensitivity, two-beam interferometry.

\section{INTRODUCTION}

$\mathbf{S}$ USPENDED core fibers are microstructured fibers with a tiny core suspended by extremely fine glass bridges. The intention of such structures is to increase the overlapping field integral of the evanescent wave, which makes the fiber attractive for a new generation of evanescent field sensors [1] and fluorescence spectroscopy methods [2]. Additionally, the reduced filling factor of the guided mode shifts the zero-dispersion wavelength to shorter wavelengths, and makes this type of fiber interesting for white light generation [3].

One basic approach to implement fiber Bragg gratings (FBGs) in suspended core fibers is the use of germanium-doped cores and hydrogen loading [1]. The small size of the core and the proximity to the holes speed hydrogen out-diffusion by orders of magnitude compared to standard fibers, where it takes place within minutes. Out-diffusion can be prevented by sealing the end faces of the microstructured fiber [4], but standard sealing methods reduce the coupling efficiency in suspended core fibers, which is detrimental for the visibility of the FBGs during grating inscription.

To bring about photoinduced refractive index changes in pure silica fibers, high photon energies and intensities are required. Preferred inscription techniques are phase mask

Manuscript received April 24, 2009; revised July 01, 2009. First published July 31, 2009; current version published September 18, 2009. The inscription experiments were performed within the COST Action 299. This work was supported by the Thuringian Ministry of Education and Cultural Affairs within the European ERDF programme. The work of L. Fernandes was supported by the Fundação para a Ciéncia e Tecnologia (FCT) under a Ph.D. grant.

M. Becker, M. Rothhardt, S. Brückner, K. Schuster, J. Kobelke, and H. Bartelt are with the Institute of Photonic Technology, Optical Fibers and Fiber Applications, D-07745 Jena, Germany (e-mail: martin.becker@ipht-jena.de).

L. Fernandes, O. Frazão, and P. V. S. Marques are with the Institute for Systems Engineering and Computers at Coimbra-Porto, 41609-007 Porto, Portugal (e-mail: lfernandes@fc.up.pt; ofrazao@fc.up.pt; psmarques@ inescporto.pt).

Color versions of one or more of the figures in this letter are available online at http://ieeexplore.ieee.org.

Digital Object Identifier 10.1109/LPT.2009.2028309

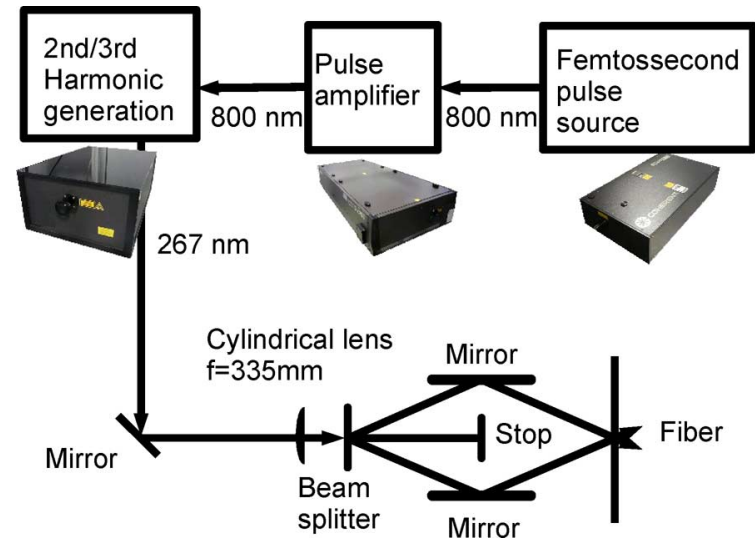

Fig. 1. Setup for two-beam interference with UV femtosecond laser pulses for FBG inscription.

inscription with IR femtosecond laser sources [5] and 193-nm argon-fluoride (ArF) excimer lasers [6], [7]. Inscription with deep UV (DUV) exposure allows a minimization of structural dimensions [8], and phase mask inscription with 267-nm femtosecond lasers [9] can also be applied to pure silica fibers [10] [11]. In this case, the fiber is placed in close proximity behind a phase mask. This requires that the photosensitivity of the target must be higher than that of the phase mask; otherwise, one risks the degradation, which comes along with a red luminescence caused by nonbridging oxygen hole centers (NBOHCs) [12], [13]. This problem can be overcome by increasing the space between phase mask and target [14].

\section{EXPERIMENTAL SETUP}

To create the standing wave for FBG inscription, a Talbot interferometer setup [15], [16] was chosen. In comparison to phase mask inscription, it offers, on the one hand, wavelength versatility of the Bragg grating reflection wavelength. On the other hand, it allows the distance between phase mask and target fiber to be increased and radiation exposure onto the phase mask to be kept at a low intensity level. The length of the DUV femtosecond pulses was measured to be below $100 \mu \mathrm{m}$. While using the interferometer and femtosecond radiation, one has to take care that pulses arrive simultaneously at the fiber with parallel pulse fronts that restrict wavelength versatility [17].

The scheme of the inscription setup is shown in Fig. 1: the driving laser system comprises a master oscillator (Coherent Mantis) and a femtosecond amplifier (Coherent Legend Elite). The IR beam $(3.6 \mathrm{~W}$ at $800 \mathrm{~nm}, 130 \mathrm{fs}, 1 \mathrm{kHz})$ passes a frequency tripler $(650 \mathrm{~mW}$ at $267 \mathrm{~nm})$, and is focused onto the fiber by a silica cylindrical lens of focal length $f=335 \mathrm{~mm}$, 


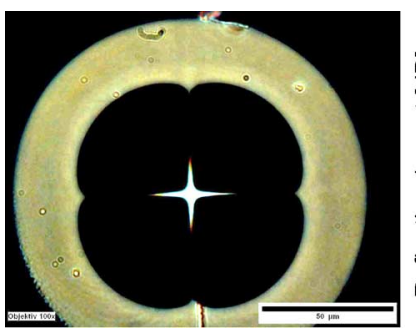

(a)

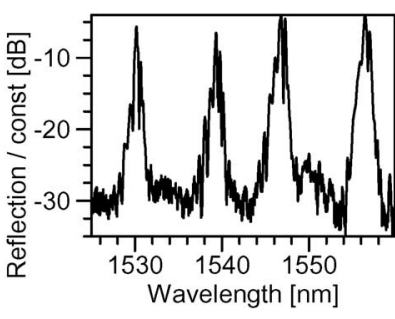

(b)
Fig. 2. Target fiber IPHT-256b1 with grating arrays. (a) Microscope picture. The black bar is the scale for $50 \mu \mathrm{m}$. (b) Reflection spectrum of a fiber Bragg grating array with four gratings.

TABLE I

FIBERS USED IN THE INSCRIPTION EXPERIMENTS

\begin{tabular}{|c|c|c|c|c|}
\hline Name & $\begin{array}{c}\text { Core } \emptyset \\
{[\mu \mathrm{m}]}\end{array}$ & $\begin{array}{c}\text { Hole } \emptyset \\
{[\mu \mathrm{m}]}\end{array}$ & $\begin{array}{c}\text { Cladding } \emptyset \\
{[\mu \mathrm{m}]}\end{array}$ & $\begin{array}{c}\text { Bridge width } \\
{[\mu \mathrm{m}]}\end{array}$ \\
\hline \hline IPHT256b1 & 5.0 & 92 & 135 & 1.5 \\
\hline IPHT256b2 & $\begin{array}{c}4.9 \\
\text { (core-hole) }\end{array}$ & 41 & 106 & 1.0 \\
\hline IPHT256b5 & 7.2 & 57 & 123 & 0.9 \\
\hline
\end{tabular}

All fibers were manufactured at the IPHT Jena.

which is placed close to the phase mask. The phase mask is optimized for $267.2 \mathrm{~nm}$ and has a grating period of $1065.3 \mathrm{~nm}$. The measured total transmission efficiency of the interferometer comprising lens, phase mask, and deflecting mirrors are $18 \%$ and $20 \%$ for the first orders focused onto the fiber. To check the geometry of the focus, a polished preform sample with the fiber material (Heraeus F300) was placed at the fiber position and exposed to radiation for around $1 \mathrm{~h}$. During exposure, a clear red luminescence was observed. The exposed region was examined with a microscope by transmitted light to make photoinduced striae visible [Fig. 3 (inset)]. This image allows estimation of the spot size to be around $0.28 \mathrm{~mm} \times 6.77 \mathrm{~mm}$. Although the impact of the irradiation in the probe is not completely homogeneous, we assume the focus profile to be nearly of top hat instead of Gaussian shape. The tripler stretches the pulse length [17] to assumably around $350 \mathrm{fs}$. Neglecting the interference fringes, we get a power density of $47 \mathrm{GW} / \mathrm{cm}^{2}$ at the fiber position. Four-leaf clover-type suspended core fibers [Fig. 2(a)] were used for our Bragg grating inscription experiments. Selected fibers, manufactured at the Institute of Photonic Technology (IPHT), with different core, hole, and outer diameter sizes are listed in Table I.

Light was coupled into the suspended core fibers through splicing with an arc-discharge fusion splicer (Sumitomo F36). A proposed splicing method to couple standard single mode fibers to microstructured fibers [18] was modified to prevent the collapse of the core and the bridges.

\section{FBG INSCRIPTION}

Following [19], we assumed that the angular orientation does not substantially affect the grating growth in the applied suspended core fibers, and there was no control of the fiber rotation angle. The inscription time was chosen to be around $30 \mathrm{~min}$ when the grating growth stopped. Longer exposure times did not result in higher Bragg grating reflections. None of the gratings

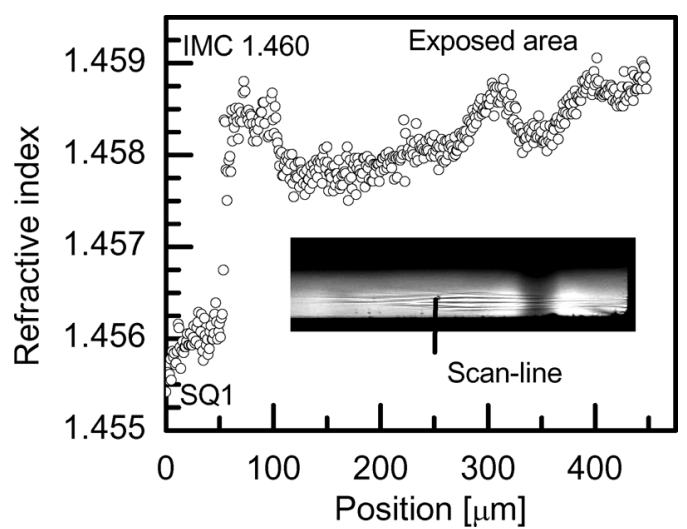

Fig. 3. Refracted near-field measurement of the index increase of the exposed region. (Inset) Regular microscope image with an indicated scan line. Schott glass (SQ1) and index matching liquid (IMC 1.460) serve as references.

did show a spectral top hat profile, as predicted by theory for strong and homogeneous index modulations. Additionally, we observed a shift of the main reflection peak after stopping the exposure by blocking the DUV laser beam. The amount of the wavelength shift depended on the fiber type and resulted to be between $0.2(252 \mathrm{~b} 1)$ and $1.3 \mathrm{~nm}(252 \mathrm{~b} 2)$. Considering a temperature increase as a reason for this shift with a sensitivity of $11 \mathrm{pm} /{ }^{\circ} \mathrm{C}$, one can estimate a temperature increase between $20^{\circ} \mathrm{C}$ and $120^{\circ} \mathrm{C}$ during FBG inscription.

We measured an exposed preform sample using a refracted near-field measurement system (Rinck Elektronik Jena), and Schott SQ1 glass and Cargille index matching liquid were used for calibration. The result (Fig. 3) shows a refractive index increase of $\Delta n=6 \times 10^{-4}$ at $650 \mathrm{~nm}$. The image of the exposed region [Fig. 3 (inset)] shows a nonhomogeneous impact on the sample, probably due to photoinduced striae in the cylindrical lens.

The resulting Bragg gratings in the final fiber structure could be measured in reflection only and show several peaks around the center wavelength, forming a clearly confined group [Fig. 2(b)]. Further reflections outside the group, as expected for multimode fibers, were below the noise level of the instrument.

\section{EFFective Mode FIELD RefRACTIVE INDEX}

The geometry of the interferometer can be used to calculate the effective refractive index $n_{\text {eff }}$ of the guided mode reflected by the FBG using the equation for the Talbot interferometer

$$
\sin (2 \phi+\alpha)=\frac{n_{\text {eff }} \lambda_{\text {inscr }}}{\lambda_{\text {Bragg }}}
$$

where $\phi$ is the tilt angle of the mirrors, $\alpha=\arcsin \left(\lambda_{\text {inscr }} / \Lambda_{\mathrm{pm}}\right)$, $\lambda_{\text {inscr }}$ is the wavelength of the inscription laser, and $\Lambda_{\mathrm{pm}}$ the phase mask period.

The effective mode field indexes versus wavelength are calculated with the tilt angle $\phi$ in (1), where the main reflection peak is used as Bragg reflection wavelength (results are shown in Fig. 4). For comparison, the data obtained are compared with the values for the bulk glass used for this fiber (Heraeus F300). The effective indexes for all fibers are all lower than expected for the bulk glass. This indicates a relevant field overlap with the air hole region, at least for the sample numbers $256 \mathrm{~b} 1$ and 


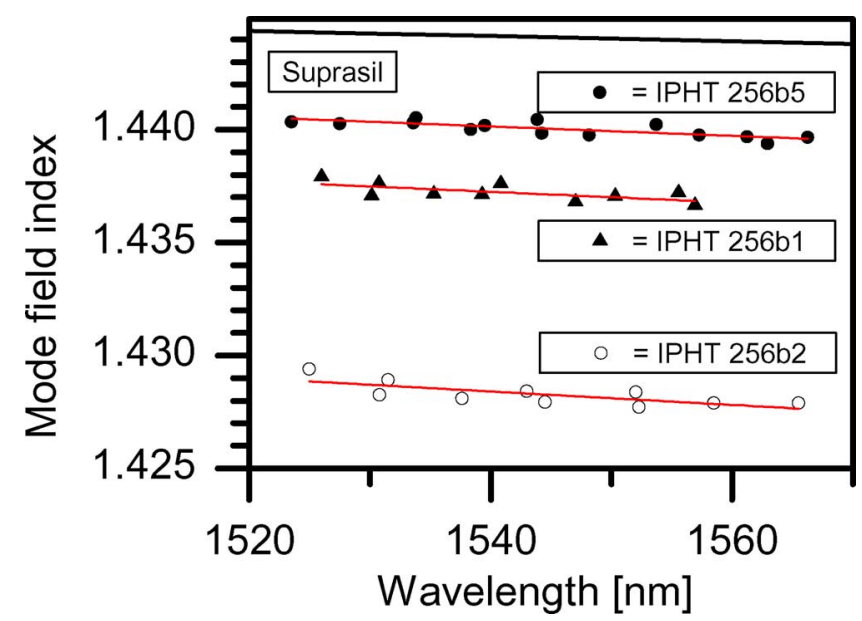

Fig. 4. Measurement of the effective mode field index of the suspended core fibers. The straight line on the top was calculated from the Sellmeier coefficients for pure silica.

TABLE II

EFFECTIVE Mode FIELd INDEXES AND FILLING FACTORS

\begin{tabular}{|c|c|c|c|c|}
\hline Name & $\begin{array}{c}\text { Mirror tilt } \phi \\
\text { for } 1550 \mathrm{~nm}\end{array}$ & $n_{\mathrm{eff}}(1550 \mathrm{~nm})$ & $\begin{array}{c}\text { Dispersion } \\
{\left[10^{-5} \mathrm{~nm}^{-1}\right]}\end{array}$ & $f(1550 \mathrm{~nm})$ \\
\hline \hline $\mathrm{SiO} 2$ & & 1.444 & -1.2 & $100 \%$ \\
\hline $256 \mathrm{~b} 5$ & $-0.079^{\circ}$ & 1.440 & $-2.1 \pm 0.4$ & $99.1 \%$ \\
\hline $256 \mathrm{~b} 1$ & $-0.096^{\circ}$ & 1.437 & $-2.4 \pm 0.9$ & $98.4 \%$ \\
\hline $256 \mathrm{~b} 2$ & $-0.140^{\circ}$ & 1.428 & $-3.0 \pm 0.9$ & $96.4 \%$ \\
\hline
\end{tabular}

256b2. To estimate the amount of light guided in the silica core, we define the confinement factor $f$ as

$$
n_{\text {eff }}=(1-f) n_{\text {air }}+f n_{\text {silica }}
$$

which is valid for step-index fibers. Using a value of $n_{\text {air }}=$ 1.000 for air, we get, with formula (2), filling factors between $96 \%$ for the fiber with core hole and $98 \%-99 \%$ for the fibers with solid cores, as listed in Table II. The dispersion of the mode field is higher than the value for pure silica.

\section{SUMMARY}

FBG arrays have been successfully implemented in pure silica suspended core fibers. The peak structure and the growth behavior differ from gratings with homogeneous grating profiles. From the resulting Bragg wavelength, one can derive the confinement factor, and therefore, the amount of overlap of the guided mode with the holes, which is an important parameter for evanescent field sensing. For different suspended core fiber types, the Talbot interferometer allows the grating reflection wavelength to be flexibly placed in the measurement range of an FBG interrogation unit.

\section{REFERENCES}

[1] M. C. P. Huy, G. Laffont, V. Dewynter, P. Ferdinand, P. Roy, J.-L Auguste, D. Pagnoux, W. Blanc, and B. Dussardier, "Three-hole microstructured optical fiber for efficient fiber Bragg grating refractometer," Opt. Lett., vol. 32, no. 16, pp. 2390-2392, 2007.

[2] S. Afshar, S. C. Warren-Smith, and T. M. Monro, "Enhancement of fluorescence-based sensing using microstructured optical fibres," Opt. Express, vol. 15, pp. 17892-17901, 2007.

[3] L. Fu, B. K. Thomas, and L. Dong, "Efficient supercontinuum generations in silica suspended core fibers," Opt. Express, vol. 16, no. 24, pp. 19629-19642, 2008.

[4] H. R. Sørensen, J. B. Jensen, F. Bruyere, and K. P. Hansen, "Practical hydrogen loading of air silica fibres," presented at the Proc. BGPP, Sydney, Australia, 2005.

[5] S. J. Mihailov, C. W. Smelser, D. Grobnic, R. B. Walker, P. Lu, H. Ding, and J. Unruh, "Bragg gratings written in all- $\mathrm{SiO}_{2}$ and Ge-doped core fibers with 800 -nm femtosecond radiation and a phase mask," $J$. Lightw. Technol., vol. 22, no. 1, pp. 94-100, Jan. 2004.

[6] N. Groothoff, J. Canning, E. Buckley, K. Lyttikainen, and J. Zagari, "Bragg gratings in air-silica structured fibers," Opt. Lett., vol. 28, no. 4, pp. 233-235, 2003.

[7] B.-O. Guan, D. Chen, Y. Zhang, H.-J. Wang, and H.-Y. Tam, "Bragg gratings in pure-silica polarization-maintaining photonic crystal fiber," IEEE Photon. Technol. Lett., vol. 20, no. 23, pp. 1980-1982, Dec. 1, 2008.

[8] M. Dubov, V. Mezentsev, I. Bennion, and D. N. Nikogosyan, "UV femtosecond laser inscribes a $300 \mathrm{~nm}$ period nanostructure in a pure fused silica," Meas. Sci. Technol., vol. 18, pp. L15-L17, 2007.

[9] A. Dragomir, D. N. Nikogosyan, K. A. Zagorulko, P. G. Kryukov, and E. M. Dianov, "Inscription of fiber Bragg gratings by ultraviolet femtosecond radiation," Opt. Lett., vol. 28, no. 22, pp. 2171-2173, 2003.

[10] K. A. Zagorulko, P. G. Kryukov, Y. V. Larionov, A. A. Rybaltovsky, and E. M. Dianov, "Fabrication of fiber Bragg gratings with $267 \mathrm{~nm}$ femtosecond irradiation," Opt. Express, vol. 12, pp. 5996-6001, 2004.

[11] L. Fu, G. D. Marshall, J. A. Bolger, P. E. Steinvurzel, E. C. Mägi, M. J. Withford, and B. J. Eggleton, "Femtosecond laser writing Bragg gratings in pure silica photonic crystal fibres," Electron. Lett., vol. 41, no. 11, pp. 638-640, May 2005.

[12] M. G. Sceats, G. R. Atkins, and S. B. Poole, "Photolytic index changes in optical fibers," Annu. Rev. Mater. Sci., vol. 23, pp. 381-410, 1993.

[13] L. Skuja, "Optically active oxygen-deficiency-related centers in amorphous silicon dioxide," J. Non-Cryst. Solids, vol. 239, pp. 16-48, 1998.

[14] M. Livitziis and S. Pissadakis, "Bragg grating recording in low-defect optical fibers using ultraviolet femtosecond radiation and a doublephase mask interferometer," Opt. Lett., vol. 33, no. 13, pp. 1449-1451, 2008.

[15] M. L. Dockney, S. W. James, and R. P. Tatam, "Fibre Bragg gratings fabricated using a wavelength tuneable laser source and a phase mask based interferometer," Meas. Sci. Technol., vol. 7, pp. 445-448, 1996.

[16] C. Li, Y.-M. Zhang, X.-F. Tian, and B.-H. Xiong, "Study of wedgeadjusted Talbot interferometer for writing fiber gratings with variable inscribed Bragg wavelengths," Opt. Eng., vol. 42, pp. 3452-3455, 2003.

[17] M. Becker, J. Bergmann, S. Brückner, M. Franke, E. Lindner, M. Rothhardt, and H. Bartelt, "Fiber Bragg grating inscription combining DUV sub-picosecond laser pulses and two-beam interferometry," Opt. Express, vol. 16, no. 23, pp. 19169-19178, 2008.

[18] Y. Wang, H. Bartelt, S. Brückner, J. Kobelke, M. Rothhardt, K. Mörl, W. Ecke, and R. Willsch, "Splicing Ge-doped photonic crystal fibers using commercial fusion splicer with default discharge parameters," Opt. Express, vol. 16, no. 10, pp. 7258-7263, 2008.

[19] T. Geernaert, T. Nasilowski, K. Chah, M. Szpulak, J. Olszewski, G. Statkiewicz, J. Wojcik, K. Poturaj, W. Urbanczyk, M. Becker, M. Rothhardt, H. Bartelt, F. Berghmans, and H. Thienpont, "Fiber Bragg gratings in germanium-doped highly birefringent microstructured optical fibers," IEEE Photon. Technol. Lett., vol. 20, no. 8, pp. 554-556, Apr. $15,2008$. 